\title{
Acute respiratory distress syndrome in an 11-month- old girl leading to extracorporeal membrane oxygenation
}

\author{
ARIJAN VERBIĆ ${ }^{1}$, KRISTINA LAH TOMULIĆ ${ }^{1}$, NEVEN FRLETA ${ }^{1}$, IGOR MEDVED ${ }^{2}$, JADRANKO SOKOLIĆ ${ }^{3}, \check{Z L L J K O ~ Z ̌ U P A N ~}^{3}$ \\ ${ }^{1}$ Intensive Care Unit, Department of Paediatrics, Clinical Hospital Centre Rijeka, Croatia \\ ${ }^{2}$ Department of Cardiac Surgery, Clinical Hospital Centre Rijeka, Croatia \\ ${ }^{3}$ Department of Anaesthesiology and Intensive Care, Clinical Hospital Centre Rijeka, Croatia
}

Corresponding author:

Arijan Verbic, Department of Paediatrics, Clinical Hospital Centre Rijeka

Istarska 43, 51000 Rijeka, Croatia

Phone: 0038551659172 , Fax: 0038551659132

E-mail: arijan.verbic@gmail.com

\section{ABSTRACT}

Acute respiratory distress syndrome (ARDS) is a condition recognised for almost fifty years, and is related to high morbidity and mortality in children. From its recognition, medical experts tried to make joint efforts to make recommendations and optimize treatment in children and adult population. The new definition of ARDS suggests treatment by introducing three levels of severity, according to $\mathrm{PaO} 2 /$ $\mathrm{FiO} 2$ and positive end-expiratory pressure. Lung-protective ventilation remains crucial in achieving better outcome in paediatric acute respiratory distress syndrome (PARDS), but promising therapies based on paediatric studies include non-invasive ventilation, endotracheal surfactant, highfrequency oscillatory ventilation (HFOV), and use of ECMO as "rescue" therapy. Nevertheless, PARDS is a real challenge for the paediatric critical care provider, and even if current state-of-the-art treatment methods are delivered, this disease often leads to fatal outcome.

We report the case of an 11-month-old female infant who developed ARDS, was treated by current up-to-date treatment methods, including ECMO, and despite this, succumbed to her illness. With this case report we would like once more to bring to consideration the current knowledge on etiology, epidemiology, diagnosis and treatment of ARDS in children, and emphasize the high morbidity and mortality related to this syndrome.

Key words: Acute respiratory distress syndrome (ARDS), children, paediatric, extracorporeal membrane oxygenation (ECMO)

\section{INTRODUCTION}

Acute respiratory distress syndrome (ARDS) is a permeability pulmonary oedema, characterized by increased permeability of pulmonary capillary endothelial cells and

alveolar epithelial cells, leading to hypoxemia that is refractory to usual oxygen therapy. (1)

The incidence of ARDS in the overall paediatric population is relatively low, with estimates ranging between 2.9 and 9.5 cases/100 000 children per year. (2) The new Berlin definition of ARDS has been published recently. This definition has suggested severity-oriented respiratory treatment by introducing three levels of severity (mild, moderate, and severe) according to $\mathrm{PaO} 2 / \mathrm{FiO} 2$. A positive end-expiratory pressure (PEEP) with a minimum of $5 \mathrm{~cm}$ $\mathrm{H} 2 \mathrm{O}$ is also required. By using the Berlin Definition instead of the American-European Consensus Conference (AECC), stages of mild, moderate, and severe ARDS were associated with increased mortality (27\%; $32 \%$; and $45 \%$ respectively) and increased median duration of mechanical ventilation in survivors (5 days; 7 days; and 9 days respectively). (3) In treatment strategies, lung-protective ventilation is still crucial in achieving better outcome in ARDS. Open-lung ventilation strategy, characterized by sufficient positive endexpiratory pressure to avoid atelectasis, a tidal volume that is limited to less than 5-7 $\mathrm{ml} / \mathrm{kg}$ per breath and a plateau pressure of $30 \mathrm{~cm}$ of water or less, provides the greatest likelihood of survival and minimizes lung injury. (2) Regarding pulmonary-specific ancillary and non-pulmonary treatment, there are a few suggested options proposed by The Paediatric Acute Lung Injury Consensus Conference Group (PALICC): 1) inhaled nitric oxide (iNO) for patients with documented pulmonary hypertension or severe right ventricular dysfunction; 2) maintaining a clear airway with cautions suctioning, 3) prone positioning for selected patients in cases of severe paediatric acute respiratory syndrome (PARDS), 4) effective targeted sedation and neuromuscular blockade (in selected cases), 5) enteral nutrition when tolerated, 6) goal-directed fluid management-fluid balance titrated to maintain adequate intravascular volume while aiming to prevent positive fluid balance, and 7) blood transfusion with haemoglobin concentration up to $7.0 \mathrm{~g} / \mathrm{dL}$, as a trigger for $\mathrm{RBC}$ transfusion in clinically stable children. The well-known treatment with exogenous surfactant and corticosteroids, and some of the newly proposed possibilities, such as helium-oxygen mixture, inhaled or IV prostaglandins therapy, plasminogen activators, fibrinolytics, inhaled $\beta$-adrenergic receptor agonists or ipratropium, IV $\mathrm{N}$-acetylcysteine, dornase alpha outside of the cystic fibrosis population, and cough-assist devices - all these have not shown clearly their benefits.

In case of failure of conventional treatment, ECMO is currently the last resort, and the key to successful survival is the implementation of ECMO before irreversible organ injury develops. ECMO should be considered to support children with severe ARDS where the cause of the respiratory failure is believed to be reversible, or the child is likely to be suitable for consideration for lung transplantation. (4) Extracorporeal Life Support Organization (ELSO) annual 
reports of ECMO use to support paediatric patients with respiratory failure is 300-500 cases/year over last 5 years. (5)

Recent advances in technology include: refinement of double lumen veno-venous (VV) cannulas, to support a large range of patient size, pumps with lower prime volumes, more efficient oxygenators, changes in circuit configuration to decrease turbulent flow and haemolysis. Veno-arterial (VA) mode of support remains the predominant type used; however, VV support has lower risk of central nervous injury and mortality. (6) Despite that, mortality has remained relatively unchanged overall (43\%) over the past several decades. Risk factors associated with death include prolonged use of mechanical ventilation $(>2$ wk) prior to ECMO, use of VA ECMO, older patient age, prolonged ECMO support and complications during ECMO. (7) Even with current state-of-the-art treatment regimens and the introduction of PARDS recommendation and protocols including ECMO, overall mortality rates range between 22 and $35 \%$. (2) It is clear that PARDS remains a heavy burden on critical care providers and hospitals dealing with it, due to high morbidity and mortality related to this syndrome.

\section{CASE REPORT}

Eleven months old female infant was admitted to our hospital because of breathing difficulties (tachypnea, dyspnea, and low oxygen saturation) due to bronchiolitis. Epidemiological history was positive for upper respiratory tract infection. Personal history revealed that she had been prenatally diagnosed with omphalocele. She was born in the 37th week of gestation by caesarean section and immediately transferred to our hospital for treatment. The infant initially received conservative treatment, which was complicated with difficulties in feeding, stool passage, weight gain and prolonged need for oxygenotherapy. Gradually normal enteral intake and stool passage were established, with normalisation in respiratory status, and she was discharged with regular follow-ups.

At the age of 7 months an operation was performed (occlusion of omphalocele), after which she had been on mechanical ventilation for two days, with uneventful postoperative period. In follow-up the child was well, without any symptoms, and regularly controlled for small ASD and mild stenosis of the left branch of the PA by paediatric cardiologists. She did not receive any chronic therapy, her vaccination was delayed by primary care paediatrician, and she did not meet the criteria for RSV immunoprophylaxis.

The girl was admitted to the pulmology department for initial symptomatic treatment. Laboratory tests and microbiological findings pointed to viral etiology of bronchiolitis (Influenza A and RSV +). After eight hours she developed acute hypercapnic respiratory failure, with impaired consciousness, and was transferred to the paediatric intensive care unit. Radiogram of thoracic organs showed bilateral infiltrates with substantial elevation of inflammatory parameters in blood exams. She was intubated, conventionally mechanically ventilated and empirical antibiotic therapy with ceftriaxone and azitomycin was introduced. $\mathrm{PaO} 2 / \mathrm{FiO} 2$ ratio of 205 and oxygenation index (OI) of 4.8 were calculated, which was compatible with a mild form of ARDS. After initial stabilization, on the second day there was further deterioration of respiratory status. There was radiologic progression of diffuse infiltrates, with $\mathrm{PaO} 2 / \mathrm{FiO} 2$ ratio of 73.75 and OI of 16.27 that were compatible with severe ARDS. Further intensive care treatment was conducted according to the current recommendations- lung protective ventilation strategy, prone positioning with lung recruitment manoeuvres, cautious negative fluid balance, endotracheal application of exogenous surfactant and finally HFOV. Trophic enteral nutrition and continuous parenteral nutrition were delivered. After a few hours, cardiac ultrasound revealed signs of pulmonary hypertension and right sided heart failure, so iNO treatment started, together with inotropes and neuromuscular blockade. Each of the treatment efforts resulted only with transient stabilization and short term positive effect on $\mathrm{PaO} 2 / \mathrm{FiO} 2$ ratio and OI. Respiratory acidosis persisted, with inadequate ventilation and oxygenation, so the need for ECMO as rescue therapy was indicated.

The child was first placed on veno-venous ECMO, which failed to achieve an adequate flow rate. Due to complications, the child was reanimated on several occasions. Opened chest operation had to be done, with placement of the central cannula (right atrium and aorta ascendens) and establishment of veno-arterial ECMO. With the adequate volume resuscitation, blood derivatives, inotrope and vasopressor support, circulatory stability and the desired flow rates were achieved. Brain ultrasound showed normal cerebral blood flow.

Despite that, the child developed signs of multiple organ failure; liver failure was followed by coagulation disorder that was corrected with FFP. Acute renal failure with signs of volume overload also developed, with the need for continuous venovenous haemodialysis (CVVHD). With V-A ECMO and CVVHD hemodynamic and respiratory stability has been finally achieved. The anticoagulant prophylaxis on ECMO was maintained in accordance to the protocol and daily open chest surgical controls were performed. Ventilation setting was set in terms of ultra-protective ventilation. Bronchoalveolar lavage with a diluted surfactant suspension and surfactant instillation was applied once more, but without any improvement in the status of the lung. By close monitoring of the neurological status, dilated pupils with no reaction to light were noticed, with abnormal EEG recording. Brain CT scan showed diffuse intracerebral hematoma with ventricular system compression, small subfalcine herniation, and signs of diffuse brain oedema, which was treated by conservative ICU treatment protocols. After 3 weeks of active treatment, the child became extremely hemodynamically unstable and despite the treatment with ECMO, CVVHD and maximum dose of vasopressor and inotrope support, death occurred.

\section{CONCLUSION}

ARDS in children is a condition whose etiology, epidemiology, diagnosis and treatment have been in focus of critical care medicine for many years. Every day novel and promising therapies are proposed by different paediatric clinical studies, including ECMO as „rescue“ therapy. ECMO was first instituted in neonatal population over 30 years ago, but even with its development and advances in technology, the mortality and morbidity remains unacceptably high. With our case report we once more emphasize the need for further investigation and institution of novel effective therapies of this syndrome. 


\section{REFERENCES}

1. Koh Y: Update in acute respiratory distress syndrome. Journal of Intensive Care 2014, 2:2

2. Cornfield DN et al.: Acute respiratory distress syndrome in children: physiology and management. Curr Opin Pediatr. 2013 Jun;25(3):338-43

3. Ranieri VM, Rubenfeld GD, Thompson BT, et al: Acute respiratory distress syndrome: The Berlin definition. JAMA 2012; 307:25262533

4. The Pediatric Acute Lung Injury Consensus Conference Group: Pediatric Acute Respiratory Distress Syndrome: Consensus Recommendations From the Pediatric Acute Lung Injury Consensus Conference. Pediatr Crit Care Med. 2015 Feb 2

5. Extracorporeal Life Support Organization. Available from: URL: http: //www.elsonet.org. Accessed 03-19-2013

6. Maslach-Hubbard A, Bratton SL: Extracorporeal membrane oxygenation for pediatric respiratory failure: History, development and current status. World J Crit Care Med 2013 November 4; 2(4): 29-39

7. Zabrocki LA, Brogan TV, Statler KD, Poss WB, Rollins MD, Bratton SL. Extracorporeal membrane oxygenation for pediatric respiratory failure: Survival and predictors of mortality. Crit Care Med 2011; 39: 364-370 